\title{
Open system dynamics from thermodynamic compatibility
}

\author{
Roie Dann $\mathbb{1}^{*}$ and Ronnie Kosloff $\mathbb{0}^{\dagger}$ \\ The Institute of Chemistry, The Hebrew University of Jerusalem, Jerusalem 9190401, Israel
}

(Received 8 January 2021; accepted 18 March 2021; published 1 April 2021)

\begin{abstract}
Thermodynamics entails a set of mathematical conditions on quantum Markovian dynamics. In particular, strict energy conservation between the system and environment implies that the dissipative dynamical map commutes with the map of the system's unitary evolution. Employing spectral analysis, we prove the general form of the ensuing master equation. The obtained structure extends thermodynamical considerations to dynamical processes. Comparing this form with master equations obtained from microscopic derivations allows validating their compatibility with thermodynamics. It predicts that coherence is not generated spontaneously under steady-state transport. Moreover, for a bipartite system-environment it singles out the global master equation as the thermodynamically compatible choice for nondriven systems, as well as supplying insight into the validity of the secular approximation.
\end{abstract}

DOI: 10.1103/PhysRevResearch.3.023006

\section{INTRODUCTION}

The generic dynamics of an open quantum system directs the system towards a thermal equilibrium state. Theoretically, this phenomenon is described within a physical theory which constitutes a dialog between quantum mechanics and thermodynamics. As most physical theories, such a theory should be formulated in terms of a set of postulates. Our present goal is to identify a minimal set of postulates, based on both thermodynamics and quantum mechanics principles, and infer the system dynamics from the axiomatic description.

The physical world of an open system is embedded within a composite system including the environment as well, which is described within an extended Hilbert space [1,2]. The composite dynamics are unitary and are generated by the global Hamiltonian

$$
\hat{H}=\hat{H}_{S}+\hat{H}_{S E}+\hat{H}_{E},
$$

where $\hat{H}_{S}$ and $\hat{H}_{E}$ are the system and environment free Hamiltonians, with an interaction term $\hat{H}_{S E}$.

Two possible approaches to obtain the reduced description of the system have been put forward. The first follows a microscopic derivation, employing a series of approximations. There are many variants of the microscopic derivation, which differ by the approximations employed, their range of validity, and specific physical models [1-24]. The original microscopic derivations were based on either a collision model [25] or the weak coupling limit, introduced by Redfield [26] and

\footnotetext{
*roie.dann@mail.huji.ac.il

†kosloff1948@gmail.com
}

Published by the American Physical Society under the terms of the Creative Commons Attribution 4.0 International license. Further distribution of this work must maintain attribution to the author(s) and the published article's title, journal citation, and DOI.
Wangsness and Bloch [27]. Compatibility with thermodynamics has been shown in the weak coupling derivation of Davies [9] as well as by Dümcke for the collision model [28]. However, the validity of this approach is restricted by the approximations conducted.

An alternative approach, which we follow here, is axiomatic. The formalism originated in the seminal work of Gorini, Kossakowski, Lindblad, and Sudarshan (GKLS) leading to the general form of a Markovian master equation [29,30]. The GKLS equation has been intensively employed; nevertheless, it does not guarantee consistency with thermodynamics. We show that by adding postulates to the ones employed to obtain the GKLS structure [30], compatibility with thermodynamic principles is guaranteed.

The postulates are as follows:

Postulate 1. The dynamical map is a completely positive trace-preserving $(C P T P)$. A thermodynamic idealization considers a large stationary thermal environment. This observation has been translated to Postulates 1 and 2; first, initially the system and environment are uncorrelated, leading to a CPTP map [31]

$$
\begin{aligned}
\hat{\rho}_{S}(t) & =\Lambda_{t}\left[\hat{\rho}_{S}(0)\right] \\
& =\operatorname{tr}_{E}\left(\hat{U}(t, 0) \hat{\rho}_{S}(0) \otimes \hat{\rho}_{E}(0) \hat{U}^{\dagger}(t, 0)\right) .
\end{aligned}
$$

Here, $\operatorname{tr}_{E}$ signifies the partial trace over the environment degrees of freedom, and $\hat{U}$ is the evolution operator of the composite system, generated by Eq. (1).

The CPTP map is contracting, meaning that the distance between any two states decreases: $\mathcal{S}\left(\hat{\rho}_{S} \| \hat{\rho}_{S}^{\prime}\right) \geqslant$ $\mathcal{S}\left(\Lambda_{t} \hat{\rho}_{S} \| \Lambda_{t} \hat{\rho}_{S}^{\prime}\right)$, where $\mathcal{S}$ is the relative entropy (or KullbackLeibler divergence) [32]. Repeated application of the map, such as in the Markovian case (Postulate 4), leads the system state towards a fixed point. This behavior is in accordance with the second law of thermodynamics, which implies that the system monotonically approaches the steady state. 
Postulate 2. The environment is initially in a stationary state with respect to the environment's free Hamiltonian $\hat{H}_{E}$. In the thermodynamic limit, we can extend this condition to all times.

Postulate 3. The fixed point of the dynamical map is a thermal state. This postulate is related to the zeroth law, implying that the map has a single fixed point $\hat{\rho}_{S}^{e q}$. Invariance of $\hat{\rho}_{S}^{e q}$ and the contraction property then imply that successive applications of the map will monotonically lead to equilibrium [33],

$$
\mathcal{S}\left(\hat{\rho}_{S} \| \hat{\rho}_{S}^{e q}\right) \geqslant \mathcal{S}\left(\Lambda_{t} \hat{\rho}_{S} \| \hat{\rho}_{S}^{e q}\right) .
$$

Postulate 4. The dynamical map $\Lambda_{t}$ is Markovian. This postulate originates from the idealization that the environment remains stationary. It is physically motivated by the separation of timescale between a sluggish system and a fast environment. Mathematically, this means that the map satisfies the semigroup property $\Lambda_{t}=\Lambda_{t-s} \Lambda_{s}$, which in turn allows us to express the map in terms of the dynamical semigroup generator $\Lambda_{t}=e^{\mathcal{L} t}[1,34-36]$. The differential form of the latter relation gives the master equation

$$
\frac{d}{d t} \hat{\rho}_{S}(t)=\mathcal{L}\left[\hat{\rho}_{S}(t)\right]
$$

Postulates 1 and 4 suffice to show that the GKLS form is the most general mathematical structure of the generator of the quantum dynamical semigroup [29,30].

Postulate 5. Strict energy conservation. A thermodynamic idealization assumes that energy does not remain trapped in the interface between the system and environment. Hence any change in the energy of the system is mirrored by a reverse change in the environment. This condition can be formulated by the relation

$$
\left[\hat{H}_{S E}, \hat{H}_{S}+\hat{H}_{E}\right]=0,
$$

commonly termed strict energy conservation (SEC) [37]. This idealization is compatible with the thermodynamic limit, in which the interface energy is negligible with respect to bulk energy. An immediate consequence of Postulate 4 is that any state which is of the form $f\left(\hat{H}_{S}+\hat{H}_{E}\right)$ is an invariant of the global dynamics [37-39]. When the environment remains in a stationary and thermal state $\hat{\rho}_{E}^{t h}=Z_{E}^{-1} e^{-\beta \hat{H}_{E}}$ throughout the dynamics (a specific case of Postulate 2), the global invariant must be $\hat{\rho}^{\text {th }}=Z^{-1} e^{-\beta\left(\hat{H}_{S}+\hat{H}_{E}\right)}$, which is consistent with the zeroth law. In turn, the associated reduced system state is $\operatorname{tr}_{E}\left(\hat{\rho}^{\text {th }}\right)=\hat{\rho}_{S}^{\text {th }}$, which motivates Postulate 3 .

The question arises, Under these postulates, what is the general structure of the dynamical equations of motion? We will show that the SEC together with the other postulates impose further restrictions on the structure of the GKLS form, following with a discussion of the physical relevance of these postulates. A primary result of this study is that the SEC along with an initial stationary environment imply that the dynamical map of the open system $\Lambda_{t}$ commutes with the isolated map $\mathcal{U}_{S}$. As a result, the generators commute as well. In turn, this sets the Lindblad jump operators of the master equation, and the thermal fixed point of the map imposes that the kinetic coefficients satisfy detailed balance.

\section{RESTRICTED STRUCTURE OF THE DYNAMICAL MAP}

The restrictions imposed on the structure of the master equation are obtained from the spectral properties of the dynamical map and its generator. The analysis relies on the following theorem:

Theorem 1. Let $\hat{H}$ [Eq. (1)] be the time-independent Hamiltonian of the composite system, with $\left[\hat{H}_{S E}, \hat{H}_{S}+\hat{H}_{E}\right]=$ 0 , and let the initial state $\hat{\rho}_{E}(0)$ be a stationary state of $\hat{H}_{E}$; then the dynamical maps $\Lambda_{t}$, Eq. (2), and $\mathcal{U}_{S}\left[\hat{\rho}_{S}(0)\right]=$ $\hat{U}_{S}(t, 0) \hat{\rho}_{S}(0) \hat{U}_{S}^{\dagger}(t, 0)$ commute:

$$
\mathcal{U}_{S}\left[\Lambda\left[\hat{\rho}_{S}\right]\right]=\Lambda\left[\mathcal{U}_{S}\left[\hat{\rho}_{S}\right]\right]
$$

where $\hat{U}_{S}(t, 0)=e^{-i \hat{H}_{S} t / \hbar}$ is the free propagator of the system and $\hat{U}(t, 0)=e^{-i \hat{H} t / \hbar}$.

The proof is based on the Kraus decomposition of the dynamical map and relies on the fact that the environment is initially stationary and the SEC condition. The detailed proof is presented in Appendix A. We emphasize that Theorem 1 applies even for non-Markovian dynamics [40], arbitrary coupling, and a wide range of environments of any size. An analogous theorem has been obtained $[41,42]$ in quantum thermodynamic resource theory for a thermal operation [37-39,43,44].

For Markovian dynamics the theorem implies that the generators also commute

$$
\mathcal{H}_{S}\left[\mathcal{L}\left[\hat{\rho}_{S}\right]\right]=\mathcal{L}\left[\mathcal{H}_{S}\left[\hat{\rho}_{S}\right]\right],
$$

with $\mathcal{H}_{S}\left[\hat{\rho}_{S}(t)\right]=-i \hbar^{-1}\left[\hat{H}_{S}, \hat{\rho}_{S}(t)\right]$. Such generators and the associated maps are termed superoperators, as they map operators to operators. Mathematically, this means that their domain is the Hilbert-Schmidt space [20,45-50], which is defined as the vector space of all system operators $\{\hat{X}\}$ endowed with an inner product $\left(\hat{X}_{i}, \hat{X}_{j}\right) \equiv \operatorname{tr}\left(\hat{X}_{i}^{\dagger} \hat{X}_{j}\right)$. This property allows us to invoke the spectral properties of vectors in Hilbert space, implying that when $\mathcal{H}_{S}$ and $\mathcal{L}$ commute, they share a common set of eigenoperators [8].

We now employ the spectral properties of $\mathcal{L}$ to construct the master equation in the GKLS form. We start by determining the eigenoperators of the system's free dynamics. These operators satisfy the eigenvalue type equation

$$
\mathcal{U}_{S} \hat{G}_{k}=e^{-i \lambda_{k} t} \hat{G}_{k},
$$

where $\lambda_{k}=-\lambda_{-k}$ and real, since the generator is antiHermitian.

It is convenient to classify the eigenoperators of $\mathcal{U}_{S}$ into two classes: unitary invariant and unitary noninvariant operators. The unitary invariant operators satisfy $\mathcal{U}_{S} \hat{G}_{k}=\hat{G}_{k}$ and therefore have degenerate eigenvalues. As a result, they can be spanned by the energy projection operators of $\hat{H}_{S}:\left\{\hat{\Pi}_{n}=\right.$ $|n\rangle\langle n|\}$. The unitary noninvariant operators are transition operators between energy states $\left\{\hat{F}_{n m}=|n\rangle\langle m|\right\}$, with $n \neq m$. Their corresponding eigenvalues are the Bohr frequencies between energy levels $|n\rangle$ and $|m\rangle: \lambda_{k}=\omega_{n m}=\left(\varepsilon_{m}-\varepsilon_{n}\right) / \hbar$. In the following, we replace the double index by a single index $k$ which runs over all the transition operators.

The generator can be decomposed into a "unitary" part and a dissipative part $\mathcal{L}=i \mathcal{H}+\mathcal{D}$. SEC implies that both $\mathcal{H}$ and $\mathcal{D}$ commute with $\mathcal{H}_{S}$ except for special cases [51]. As a result, the unitary part is also composed of the invariant set $\{\hat{\Pi}\}$. 
Physically, this means that the environment imposes a shift to the bare energy spectrum, commonly known as the Lamb shift $[52,53]$. The next step is to construct $\mathcal{D}$ from the same set of eigenoperators.

To simplify the analysis, we first consider the case when there is no degeneracy in the spectrum of $\mathcal{U}_{S}$ except for the unitary invariant subspace. This corresponds to an energy spectrum where no two Bohr frequencies are the same. This condition as well as relation (A1) entails that

$$
\mathcal{D}^{*}\left[\hat{F}_{k}\right] \propto \hat{F}_{k} \quad \text { and } \quad \mathcal{D}^{*}\left[\hat{\Pi}_{k}\right]=\sum_{j} a_{j} \hat{\Pi}_{j}
$$

where $\mathcal{D}^{*}$ denotes the adjoint (Heisenberg representation) of $\mathcal{D}$, and similarly for all the superoperators.

We start the derivation from the form introduced by Gorini et al. [29] (also see Ref. [2]). Utilizing Postulates 1 and 4, they obtain an equivalent form of the GKLS structure

$$
\mathcal{D}^{*}[\bullet]=\sum_{i, j=1}^{N^{2}-1} a_{i j}\left(\hat{S}_{j}^{\dagger} \bullet \hat{S}_{i}-\frac{1}{2}\left\{\hat{S}_{j}^{\dagger} \hat{S}_{i}, \bullet\right\}\right),
$$

where $\{\hat{S}\}$ constitutes an orthonormal operator basis, such that one of the basis operators is chosen to be proportional to the identity and all other operators are traceless. In addition, the coefficient matrix $a=\left[a_{i j}\right]$ is Hermitian and positive. We choose the basis $\{\hat{S}\}$ to be composed of the transition operators $\{\hat{F}\}$ and orthonormal operators from the unitary invariant subspace $\{\hat{G}\}$; these can be chosen as matrices which are proportionate to the diagonal generalized Gell-Mann matrices [54-56]. In the general form of Eq. (10), all the possible combinations of $\hat{S}_{i}, \hat{S}_{j}$ in this basis exist. However, only certain terms are compatible with Eq. (6) and the eigenoperator condition equation (9). Employing the property that $\hat{F}_{k}$ are transition operators, we can obtain the diagonal form of the GKLS equation (cf. Appendixes B-D)

$$
\begin{aligned}
\mathcal{D}^{*}[\bullet]= & \sum_{k=-M}^{M} \gamma_{k}\left(\hat{F}_{k}^{\dagger} \bullet \hat{F}_{k}-\frac{1}{2}\left\{\hat{F}_{k}^{\dagger} \hat{F}_{k}, \bullet\right\}\right) \\
& -\sum_{n=1}^{N} \lambda_{n}\left[\hat{V}_{n},\left[\hat{V}_{n}, \bullet\right]\right],
\end{aligned}
$$

where $M=N(N-1) / 2$, with $\operatorname{dim}\left(\hat{H}_{S}\right)=N, \hat{F}_{k}^{\dagger}=\hat{F}_{-k}$, and $\gamma_{k}=a_{k k}$ with $\gamma_{0}=0$. The unitary invariant operators $\{\hat{V}\}$ are linear combinations of the projection operators $\{\hat{\Pi}\}$ with real coefficients. The final restriction on the structure of $\mathcal{D}^{*}$ can be imposed by the fixed thermal state of the dynamical map (Postulate 3). The steady-state condition infers that the kinetic coefficient satisfy detailed balance $\gamma_{k} / \gamma_{-k}=e^{-\hbar \omega_{k} \beta}$ (cf. Appendix E). Detailed balance only fixes the ratio between pairs of kinetic coefficients. To completely determine their value, one can rely on a microscopic derivation which complies with the structure $[2,9,28,57]$ of Eq. (11), or on a numerical calculation. Alternatively, a different fixed state can be chosen, leading to a different ratio between the pairs of kinetic coefficients.

Overall, the dissipator [Eq. (11)] is composed of two contributions. The first sum induces transitions between eigenstates of $\hat{H}_{S}$, with probabilities that satisfy detailed balance, while the second term describes pure dephasing. Utilizing the spectral decomposition of the system Hamiltonian, the dephasing term can be expressed simply as a sum of double commutators of functions of the Hamiltonian $-\sum_{i}\left[f_{i}\left(\hat{H}_{S}\right),\left[f_{i}\left(\hat{H}_{S}\right), \bullet\right]\right]$. This is in contrast to the microscopic derivation, which originates from a perturbation treatment in the system-environment coupling, for which pure dephasing appears only beyond fourth order in the perturbation expansion [58]. Here, the pure-dephasing term emerges naturally from the unitary invariant set.

Considering the case where a degeneracy in $\{\hat{F}\}$ exists, the eigenoperators of $\mathcal{D}$ will become a linear combination of the degenerate set. As a consequence, the master equation may include cross terms of the form $\hat{F}_{i}^{r} \bullet \hat{F}_{j}^{r}$ with $i \neq j$. In this case, there is an additional degree of freedom which allows imposing further restrictions which will determine the Lindblad jump operators. For example, in a transport scenario between two baths one can impose the condition of a vanishing current in the limit of zero internal coupling [59-61]. In this scenario, it has been shown that the resonance condition leads to quantum correlations between the two baths [61]. The additional freedom in the choice of the dephasing term (either local or global) in Eq. (11) can eliminate this correlation so as to comply with the assumption of independent baths.

\section{CRITICAL ANALYSIS OF THE PHYSICAL IDEALIZATIONS}

The time-reversal symmetry breaking is the main conflict between thermodynamics and quantum mechanics. The theory of open quantum systems resolves this conflict by imposing a partition between a small system and a large environment [62]. In this paper we focus on an isothermal partition which allows heat transport, while maintaining the integrity of the system and environment. This idealization is reflected by Postulates 1 and 4 .

The validity of this idealization has to be confronted with reality. Typically, open system processes are of Markovian nature. Even for non-Markovian dynamics the common approach is to embed the system in a larger Markovian framework $[63,64]$. This motivates the a priori postulation of the semigroup property. The postulate contradicts the principle of unitary evolution; nevertheless, up to a coarse-grained timescale it fits the observed reality. In addition, the Markovian property implies that the composite state must remain separable throughout the evolution [62], which enables a local description of the system.

Our construction relies on a CPTP map which leads to a GKLS form. The complete positivity property is related to the separability of the composite state at the initial time. This assumption has been criticized as nonphysical $[65,66]$. In response to the criticism, justifications for the CPTP property have been presented $[32,67]$.

The strict energy conservation condition is also a thermodynamic idealization, leading to Eq. (7). This condition is satisfied asymptotically in the weak coupling limit and the low-density collision model [25,28]. Moreover, we will show that for Markovian dynamics, it is implicitly satisfied when the unitary and dissipative maps commute. 
In the Markovian regime, commutation of the dynamical maps is synonymous with the commutation of the corresponding generators and vice versa. Many master equations satisfy this property, for example, the GKLS master equation of Alicki, where detailed balance was imposed $[4,46]$. In addition, the popular Davies construction, which is based on the Born-Markov-secular approximation, also satisfies the commutation property $[2,9,68]$. The critical question is, do these master equations comply with the SEC condition?

Addressing this issue, we study the reverse condition: Does commutation of the generators imply the SEC condition? For Markovian dynamics, by expanding the infinitesimal maps $\Lambda_{\varepsilon}$ and $\mathcal{U}_{S}(\varepsilon, 0)$ and demanding that they commute, we obtain strict conditions on the generators. These are fulfilled naturally when the SEC condition is satisfied (cf. Appendix F for further details). The analysis also allows for some nonphysical exceptions.

\section{DISCUSSION}

The discipline of open quantum systems faces the challenge of assessing the multitudinous microscopic derivations. Different subfields have varying traditions leading to different variants of the master equation [1-24]. There is a need for both unification and classification of validity of the various approaches.

A major line of research approached this problem by constructing analytical models and comparing them to numerically "exact" simulations [59-61,69]. General conclusions are then inferred from a specific model. The drawback of this approach is that it heavily relies on solvable models. Almost exclusively, the chosen environment is a bosonic normal mode bath or can be shown to be equivalent to such a bath [70]. The drawback of this environment model is that its ergodic properties are weak [71]; for example, it does not fulfill the eigenvalue thermalization hypothesis [72-75]. This stems from the property that the normal model structure lacks an internal mechanism for self-equilibration. In comparison, the axiomatic approach presented in this paper addresses this issue from a broad dynamical perspective. The validity regime of the master equation is solely determined by the basic postulates and is independent of the studied model. As a consequence, we can verify the thermodynamic validity of various master equations.

In addition, it has been observed that in many cases the validity of the GKLS form exceeds the range imposed by the microscopic derivations [76]. This means that by refitting the kinetic coefficients the equations can be employed to describe physical reality accurately $[77,78]$. This observation agrees with our resulting structure [Eq. (11)].

The postulates presented here overlap with the paradigm of quantum thermodynamic resource theory [37-39,43,44]. The main difference in the approaches is the Markovian assumption (Postulate 4), which allows us to attain a dynamical theory. A second difference concerns Postulate 2, where we assume that initially the environment state is a stationary, not necessarily thermal, state.

The formal construction also sheds light on unresolved issues in the discipline of open system dynamics. Master equations are typically of either a local or global character.
The local approach assumes that the environment couples only locally to a part of the system, while in the global approach, the environment couples to global degrees of freedom of the whole system. The two approaches have been studied thoroughly, and their validity has been a subject of much debate $[59,60,78-83]$. In the construction presented the generator coincides with the global master equation, meaning that the unitary noninvariant operators $\{\hat{F}\}$, which constitute the jump operators of the master equation, are eigenoperators of the "global" system propagator and not solely of a subsystem.

The origin of this property can be traced back to the strict energy conservation condition. For instance, consider a bipartite system, composed of a part that interacts with the environment $\hat{H}_{S}^{(e)}$ and a passive part $\hat{H}_{S}^{(p)}$. When the two parts interact, the system Hamiltonian reads $\hat{H}_{S}=\hat{H}_{S}^{(e)}+\hat{H}_{S}^{(p)}+$ $\hat{H}_{S}^{(i)}$. When $\left|\hat{H}_{S}^{(i)}\right| \ll\left|\hat{H}_{S}^{(e)}\right|,\left|\hat{H}_{S}^{(p)}\right|$, it would seem appropriate to describe the dynamics by a local master equation, where the generator acts only on a part of the system. However, this construction violates strict energy conservation since the internal and external interaction terms do not commute. In contrast, a global master equation manifests strict energy conservation for the combined system. It has been shown recently that thermodynamics consistency can be restored by adding a resource, a work term to compensate for interaction [81]. For example, an additional local dephasing term on the passive system would do the job.

The role of the secular approximation has been debated [9,11,12,84-87]. Typically, this approximation is justified only when the coarse-graining time is much larger than the timescale related to the difference between the system's Bohr frequencies. However, in many physical systems, such as in many-body systems, the level spacing can decrease to a point where the secular approximation breaks down. In these cases, it is not clear how to proceed and obtain a GKLS master equation from first principles. As a consequence of the SEC condition (Postulate 5), the present construction does not restrict the magnitude of the Bohr frequencies and determines the unitary noninvariant Lindblad jump operators uniquely, as long as the Bohr frequencies remain nondegenerate. In addition, such an interaction implies that the Hamiltonian in the interaction representation relative to the bare system environment Hamiltonian is stationary.

The present analysis can be extended to transport phenomena, where the primary system is coupled to several environments characterized by different temperatures. In this case, we relax Postulate 3 and can combine different environments which are all together in a stationary state. Theorem 1 remains valid under these conditions leading to a GKLS generator (10) with kinetic coefficients that are combinations of the detailed balance coefficients of individual environments. As a result, the system steady state lacks coherence in the energy basis. This observation has been noticed in the analysis of a three-qubit absorption refrigerator [88]. In contrast, the local treatment exhibits coherence [89].

In analogy to strict energy conservation, other conserved properties could also be incorporated in the GKLS structure, such as conservation of the number of particles. This would result in a modification of the detailed balance conditions [57]. 
Our current result can also be extended to the nonMarkovian regime. The commutativity of the free and open dynamical maps holds for the general case (Theorem 1). This implies that a time-dependent generator of non-Markovian dynamics will be characterized by an equation similar to Eq. (11), where the time dependence is absorbed in the kinetic coefficients [40]. This issue needs to be addressed in a future study.

In the Markovian regime, the commutativity of the unitary and dissipative parts of the master equation infers that if there is no degeneracy in the noninvariant subspace of the unitary part, the dissipative part is diagonalizable. This leads us to conjecture that there are no exceptional points, i.e., nonHermitian degeneracies in the dissipative spectrum [90-93].

Returning to thermodynamics, the SEC condition unambiguously defines the heat current as $\dot{Q}=\left\langle\mathcal{L}^{*}\left[\hat{H}_{S}\right]\right\rangle$, since the change in energy of the system corresponds to energy transferred to the environment. This heat transport accounts for entropy production in the environment $\Delta S_{E}$ due to energy transfer. Additional entropy production is generated by loss of coherence and mutual information [94-96]. Work can be included within this framework by partitioning the "system" into a primary system and control system. Work is then identified with the energy transfer between the control and the primary system that conserves entropy. By considering a large control quantum system, the semiclassical limit leads to an extension of the current analysis, including explicitly timedependent Hamiltonians [97].

The considered postulates reflect idealization of reality and are therefore restrictive. Nevertheless, for any GKLS master equation which represents reality, we can invert the viewpoint and find an environment and interaction term which lead to the same reduced dynamics. This viewpoint may allow us to simplify the thermodynamic analysis and lead to further insights.

To summarize, the theme of this paper is the formulation of thermodynamic principles in terms of mathematical statements imposed on the quantum dynamical map. Through spectral analysis we have obtained a restricted structure of the master equation which by construction complies with thermodynamics. This structure can be employed as an independent validator of open system dynamics which are obtained by means of physical approximations.

\section{ACKNOWLEDGMENTS}

We thank Robert Alicki, Peter Salamon, James Nulton Erez Boukobza, Nimrod Moiseyev, Adi Pick, Ander Tobalina, Amikam Levy, Daniel Lidar, and Luis Correa for sharing their views and comments. This research was supported by the Adams Fellowship Program of the Israel Academy of Sciences and Humanities, the National Science Foundation under Grant No. NSF PHY-1748958, and Israel Science Foundation Grant No. 2244/14.

\section{APPENDIX A: PROOF OF THEOREM 1}

We provide a detailed proof of Theorem 1, which leads to the spectral analysis presented in the main text.

Theorem 1. Let $\hat{H}$ be the time-independent Hamiltonian of the composite system, with $\left[\hat{H}_{S E}, \hat{H}_{S}+\hat{H}_{E}\right]=0$, and let the initial state $\hat{\rho}_{E}(0)$ be a stationary state of $\hat{H}_{E}$; then the dynamical maps $\Lambda_{t}$ and $\mathcal{U}_{S}$ commute:

$$
\mathcal{U}_{S}\left[\Lambda\left[\hat{\rho}_{S}\right]\right]=\Lambda\left[\mathcal{U}_{S}\left[\hat{\rho}_{S}\right]\right]
$$

Proof. We first introduce some notations: The free propagators of the environment and composite (uncoupled) system are given by $\hat{U}_{E}(t, 0)=e^{-i \hat{H}_{E} t / \hbar}$ and $\hat{U}_{S E}(t, 0)=e^{-i\left(\hat{H}_{S}+\hat{H}_{E}\right) t / \hbar}$; moreover, the spectral decomposition of the environment Hamiltonian reads $\hat{H}_{E}=\sum_{i} c_{i}\left|\chi_{i}\right\rangle\left\langle\chi_{i}\right|$. Since the initial state of the environment is stationary with respect to $\hat{H}_{E}$, it can also be expressed as $\hat{\rho}_{E}(0)=\sum_{i} \lambda_{i}\left|\chi_{i}\right\rangle\left\langle\chi_{i}\right|$. To simplify the notation, in this proof we emit the time dependence of the propagators and maps; nevertheless, it should be clear that they induce a time translation from initial time $\left(t^{\prime}=0\right)$ to time $t^{\prime}=t$.

Utilizing the spectral decomposition of the environment's initial state, any quantum dynamical map can be expressed in a Kraus form [31]

$$
\hat{\rho}_{S}(t)=\sum_{i j} \hat{K}_{i j} \hat{\rho}_{S}(0) \hat{K}_{i j}^{\dagger}
$$

where $\hat{K}_{i j}=\sqrt{\lambda_{i}}\left\langle\chi_{j}|\hat{U}(t, 0)| \chi_{i}\right\rangle$ with $\sum_{i j} \hat{K}_{i j}^{\dagger} \hat{K}_{i j}=\hat{I}_{S}$. In the Heisenberg representation the dynamical map becomes $\hat{O}_{S}^{H}(t)=\Lambda^{*}\left[\hat{O}_{S}\right]=\sum_{i j} \hat{K}_{i j}^{\dagger} \hat{O}_{S}(0) \hat{K}_{i j}$, where the superscript $H$ and asterisk designate operators and superoperators (dynamical maps) in the Heisenberg representation and $\hat{O}_{S}$ is a system operator.

Using the Kraus representation, the product of dynamical maps is explicitly expressed as

$$
\begin{aligned}
\mathcal{U}_{S}^{*}\left[\Lambda^{*}\left[\hat{O}_{S}\right]\right] & =\hat{U}_{S}^{\dagger}\left(\sum_{i j} \hat{K}_{i j}^{\dagger} \hat{O}_{S} \hat{K}_{i j}\right) \hat{U}_{S} \\
& =\sum_{i} \lambda_{i}\left\langle\chi_{i}\left|\hat{U}_{S}^{\dagger} \hat{U}^{\dagger} \hat{O}_{S} \sum_{j}\right| \chi_{j}\right\rangle\left\langle\chi_{j}\left|\hat{U} \hat{U}_{S}\right| \chi_{i}\right\rangle \\
& =\sum_{i} \lambda_{i}\left\langle\chi_{i}\left|\hat{U}_{S}^{\dagger} \hat{U}^{\dagger} \hat{O}_{S} \hat{U} \hat{U}_{S}\right| \chi_{i}\right\rangle,
\end{aligned}
$$

where the second equality is achieved by identifying the environment identity operator $\hat{I}_{E}=\sum_{j}\left|\chi_{j}\right\rangle\left\langle\chi_{j}\right|$. Inserting the identity operator $\hat{U}_{E} \hat{U}_{E}^{\dagger}=\hat{I}_{E}$ twice, we obtain

$$
\begin{aligned}
\mathcal{U}_{S}^{*}\left[\Lambda^{*}\left[\hat{O}_{S}\right]\right] & =\sum_{i} \lambda_{i}\left\langle\chi_{i}\left|\hat{U}_{E} \hat{U}_{S E}^{\dagger} \hat{U}^{\dagger} \hat{O}_{S} \hat{U} \hat{U}_{S E} \hat{U}_{E}^{\dagger}\right| \chi_{i}\right\rangle \\
& =\sum_{i} \lambda_{i}\left\langle\chi_{i}\left|\hat{U}_{S E}^{\dagger} \hat{U}^{\dagger} \hat{O}_{S} \hat{U} \hat{U}_{S E}\right| \chi_{i}\right\rangle
\end{aligned}
$$

The second equality is obtained by utilizing the eigenvalue equation $\hat{U}_{E}\left|\chi_{i}\right\rangle=e^{-i c_{i} t / \hbar}\left|\chi_{i}\right\rangle$ for the eigenstates $\left\{\left|\chi_{i}\right\rangle\right\}$. Next, strict energy conservation implies that $\left[\hat{H}, \hat{H}_{S}+\hat{H}_{E}\right]=0$, which in turn implies that the associated propagators satisfy $\left[\hat{U}, \hat{U}_{S E}\right]=0$. This relation leads to the final form

$$
\mathcal{U}_{S}^{*}\left[\Lambda^{*}\left[\hat{O}_{S}\right]\right]=\sum_{i} \lambda_{i}\left\langle\chi_{i}\left|\hat{U}^{\dagger} \hat{U}_{S E}^{\dagger} \hat{O}_{S} \hat{U}_{S E} \hat{U}\right| \chi_{i}\right\rangle
$$


Following a similar derivation, the product in reverse order of the dynamical maps gives

$$
\begin{aligned}
\Lambda^{*}\left[\mathcal{U}_{S}^{*}\left[\hat{O}_{S}\right]\right] & =\sum_{i j} \hat{K}_{i j}^{\dagger} \hat{U}_{S}^{\dagger} \hat{O}_{S} \hat{U}_{S} \hat{K}_{i j} \\
& =\sum_{i} \lambda_{i}\left\langle\chi_{i}\left|\hat{U}^{\dagger} \hat{U}_{S E}^{\dagger} \hat{U}_{E} \hat{O}_{S} \hat{U}_{E}^{\dagger} \hat{U}_{S E} U\right| \chi_{i}\right\rangle \\
& =\sum_{i} \lambda_{i}\left\langle\chi_{i}\left|\hat{U}^{\dagger} \hat{U}_{S E}^{\dagger} \hat{O}_{S} \hat{U}_{S E} U\right| \chi_{i}\right\rangle,
\end{aligned}
$$

where the last equality stems from the commutativity of local operators of the system and environment $\left[\hat{U}_{E}, \hat{O}_{S}\right]=0$.

Finally, Eqs. (A5) and (A6) imply the desired result

$$
\mathcal{U}_{S}^{*}\left[\Lambda^{*}\left[\hat{O}_{S}\right]\right]=\Lambda^{*}\left[\mathcal{U}_{S}^{*}\left[\hat{O}_{S}\right]\right] .
$$

From the equivalence of the Schrödinger and Heisenberg representations we can infer that $\Lambda$ and $\mathcal{U}_{S}$ commute. In Hilbert-Schmidt space this relation is simply expressed as $\left[\widetilde{\mathcal{U}}_{S}, \widetilde{\Lambda}\right]=0$, where superoperators in Hilbert-Schmidt space are denoted with a superscript wide tilde.

This result is quite general, as the proof employed only Postulate 4 of strict energy conservation and the property that environment is stationary. A short proof for thermal operations $\mathcal{E}$ has been derived previously [41]. For an initial thermal environment the thermal operation coincides with the dynamical map $\Lambda_{t}$.

\section{Consequences of the commutation relation $\left[\tilde{\mathcal{U}}_{s}, \tilde{\Lambda}\right]=0$ on the semigroup generators}

If $\Lambda$ is a semigroup map, the generator is defined by $\mathcal{L}\left[\hat{\rho}_{S}\right]=\lim _{\varepsilon \rightarrow 0} \frac{1}{\varepsilon}\left(\Lambda_{\varepsilon}\left[\hat{\rho}_{S}\right]-\hat{\rho}_{S}\right)$ [35]. This definition together with Theorem 1 implies the commutativity of $\mathcal{U}_{S}$ with the generator of the dynamical map $\mathcal{L}$. We define the Lamb shift as $\mathcal{H}-\mathcal{H}_{S}$, which typically commutes with $\mathcal{H}_{S}$ and $\mathcal{D}$ [2]. Alternatively, the Lamb shift can be incorporated into $\hat{H}_{S}$, which infers that $\mathcal{H}=\mathcal{H}_{S}$. The ambiguity in the system energy results from the possibility of accounting for a part of the interface energy within the system Hamiltonian. This property and the fact that $\mathcal{H}_{S}$ is an analytical function of $\mathcal{U}_{S}$ (time-independent Hamiltonian) imply that $\mathcal{H}_{S}$ commutes with $\mathcal{D}$ and $\mathcal{L}$, or alternatively,

$$
\left[\tilde{\mathcal{H}}_{S}, \tilde{\mathcal{D}}\right]=0, \quad\left[\tilde{\mathcal{H}}_{S}, \tilde{\mathcal{L}}\right]=0 .
$$

In general, $\widetilde{\mathcal{L}}$ is non-Hermitian, which does not guarantee a spectral decomposition [49,50]. However, these relations imply that $\widetilde{\mathcal{L}}$ and $\widetilde{\mathcal{D}}$ are diagonalizable (normal).

\section{APPENDIX B: MATHEMATICAL CONSTRUCTION OF THE QUANTUM DYNAMICAL SEMIGROUP GENERATOR AND THE ORTHOGONALITY OF THE LINDBLAD OPERATORS}

This Appendix includes a summarized textbook construction of the general form of the generator $\mathcal{L}$; for further details, see Chap. 3.2 of Ref. [2], which is in the spirit of the original work of Gorini, Kossakowski, and Sudarshan [29]. We utilize a number of the intermediate results of this proof in the main derivation.
We begin by introducing an orthonormal operator basis $\{\hat{S}\}$ for the system's Liouville space. The operators of the basis are chosen such that a single operator is proportional to the identity $\hat{S}_{N^{2}}=(1 / N)^{1 / 2} \hat{I}_{S}$ and the others are traceless. Utilizing the completeness relation and the inner product in Liouville space, the Kraus operators [Eq. (A2)] can be expressed as

$$
\hat{K}_{\mu \nu}=\sum_{k=1}^{N^{2}}\left(\hat{S}_{i}, \hat{K}_{\mu \nu}\right) \hat{S}_{i} .
$$

Substituting (B1) into the dynamical map equation (A2), we obtain

$$
\Lambda_{t}\left[\hat{\rho}_{S}(0)\right]=\sum_{i, j=1}^{N^{2}} r_{i j} \hat{S}_{i} \hat{\rho}_{S}(0) \hat{S}_{j}^{\dagger},
$$

where

$$
r_{i j}=\sum_{\mu, \nu}\left(\hat{S}_{i}, \hat{K}_{\mu \nu}\right)\left(\hat{S}_{j}, \hat{K}_{\mu \nu}\right)^{*} .
$$

The coefficient matrix $r=\left[r_{i j}\right]$ can be shown to be Hermitian and positive.

Next, we introduce the following coefficients:

$$
\begin{aligned}
a_{N^{2} N^{2}} & =\lim _{\varepsilon \rightarrow 0} \frac{r_{N^{2} N^{2}(\varepsilon)}-N}{\varepsilon}, \\
a_{i N^{2}} & =\lim _{\varepsilon \rightarrow 0} \frac{r_{i N^{2}}(\varepsilon)}{\varepsilon}, \\
a_{i j} & =\lim _{\varepsilon \rightarrow 0} \frac{r_{i j}(\varepsilon)}{\varepsilon},
\end{aligned}
$$

where $i, j=1, \ldots, N^{2}-1, \quad$ and the operators $\hat{S}=$ $(1 / N)^{1 / 2} \sum_{i=1}^{N^{2}-1} a_{i N^{2}} \hat{S}_{i}, \quad \hat{R}=\frac{1}{2 N} a_{N^{2} N^{2}} \hat{l}_{S}+\frac{1}{2}\left(\hat{F}^{\dagger}+\hat{F}\right), \quad$ and $\hat{J}=\frac{1}{2 i}\left(\hat{F}^{\dagger}-\hat{F}\right)$, with $\hat{F}=\frac{1}{\sqrt{N}} \sum_{i=1}^{N^{2}-1} a_{i N^{2}} \hat{F}_{i}$.

Substituting the coefficients of Eq. (B4) into the generator of the dynamical semigroup $\mathcal{L}\left[\hat{\rho}_{S}\right]=\lim _{\varepsilon \rightarrow 0} \frac{1}{\varepsilon}\left(\Lambda_{\varepsilon}\left[\hat{\rho}_{S}\right]-\hat{\rho}_{S}\right)$ and expressing the result in terms of $\hat{S}, \hat{R}$, and the Hermitian operator $\hat{J}$, the generator can be written as

$$
\mathcal{L}\left[\hat{\rho}_{S}(t)\right]=-i\left[\hat{J}, \hat{\rho}_{S}(t)\right]+\left\{\hat{R}, \hat{\rho}_{S}(t)\right\}+\sum_{i j=1}^{N^{2}-1} a_{i j} \hat{S}_{i} \hat{\rho}_{S}(t) \hat{S}_{j}^{\dagger} .
$$

This form must preserve the trace of the density matrix $\operatorname{tr}_{S}\left(\mathcal{L}\left[\hat{\rho}_{S}(t)\right]\right)=0$, which implies that $\hat{R}=-\frac{1}{2} \sum_{i, j}^{N^{2}-1} a_{i j} \hat{S}_{j}^{\dagger} \hat{S}_{i}$. Inserting this relation into Eq. (B6) leads to the form originally obtained by Gorini, Kossakowski, and Sudarshan [29]

$$
\begin{aligned}
\mathcal{L}\left[\hat{\rho}_{S}(t)\right]= & -i\left[\hat{J}, \hat{\rho}_{S}(t)\right] \\
& +\sum_{i, j=1}^{N^{2}-1} a_{i j}\left(\hat{S}_{i} \hat{\rho}_{S}(t) \hat{S}_{j}^{\dagger}-\frac{1}{2}\left\{\hat{S}_{j}^{\dagger} \hat{S}_{i}, \hat{\rho}_{S}(t)\right\}\right) .
\end{aligned}
$$

The GKLS form is obtained by basis transformation which diagonalizes the coefficient matrix $a$. The relations defined in Eq. (B4) and the properties of the coefficient matrix $c$ imply that $a=\left[a_{i j}\right]$ is also Hermitian and positive. As a consequence, it can be diagonalized by a similarity transformation 
$u^{\dagger} a u=\operatorname{diag}\left(\lambda_{1}, \ldots, \lambda_{N^{2}-1}\right)$. Introducing a new set of operators $\{\hat{L}\}$, satisfying $\hat{S}_{i}=\sum_{k}^{N^{2}-1} u_{i k}^{*} \hat{L}_{k}$, and expressing $\mathcal{L}$ in terms of these operators leads to the final general GKLS form

$$
\begin{aligned}
\mathcal{L}\left[\hat{\rho}_{S}(t)\right]= & -i\left[\hat{J}, \hat{\rho}_{S}(t)\right] \\
& +\sum_{k} \lambda_{k}\left(\hat{L}_{k} \bullet \hat{L}_{k}^{\dagger}-\frac{1}{2}\left\{\hat{L}_{k}^{\dagger} \hat{L}_{k}, \bullet\right\}\right) .
\end{aligned}
$$

\section{APPENDIX C: RESTRICTION ON THE MASTER EQUATION}

We begin our analysis by building upon the construction of Appendix B. Equation (B7) implies that in general the dissipator (in the Heisenberg representation) can be expressed as

$$
\mathcal{D}^{*}[\bullet]=\sum_{i, j=1}^{N^{2}-1} a_{i j}\left(\hat{S}_{j}^{\dagger} \bullet \hat{S}_{i}-\frac{1}{2}\left\{\hat{S}_{j}^{\dagger} \hat{S}_{i}, \bullet\right\}\right) .
$$

As mentioned in the main text, we choose the basis $\{\hat{S}\}$ to be composed of the transition operators $\{\hat{F}\}$ and orthonormal operators from the unitary invariant subspace $\{\hat{G}\}$. We denote this basis by $\mathfrak{F}$.

The unitary invariant operators $\{G\}$ are generally a linear combination of the energy projection operators $\hat{G}_{j}=$ $\sum_{k} g_{j k} \Pi_{k}$. We can therefore express $\mathcal{D}^{*}$ in the form of Eq. (C1) with jump operators from the basis $\mathfrak{k}=\{\hat{F}\} \bigcup\{\hat{\Pi}\}$. In this basis, by expressing the transition and projection operators in terms of the eigenstates of $\hat{H}_{S}$ we obtain a sum of typical terms of the form

$$
\begin{aligned}
\mathcal{D}_{n m n^{\prime} m^{\prime}}^{*}[\bullet] & \equiv \hat{S}_{j}^{\dagger} \bullet \hat{S}_{i}-\frac{1}{2}\left\{\hat{S}_{j}^{\dagger} \hat{S}_{i}, \bullet\right\} \\
& =|n\rangle\left\langle m|\bullet| n^{\prime}\right\rangle\left\langle m^{\prime}\right|-\frac{1}{2}\left\{|n\rangle\left\langle m \| n^{\prime}\right\rangle\left\langle\left. m\right|^{\prime}, \bullet\right\}\right.
\end{aligned}
$$

for $n, m, n^{\prime}, m^{\prime} \in 1, \ldots, N$. For a general operator $|k\rangle\langle l|$ in $\mathfrak{K}$, Eq. (C2) becomes

$$
\begin{aligned}
\mathcal{D}_{n m n^{\prime} m^{\prime}}^{*}[|k\rangle\langle l|]= & \delta_{m k} \delta_{l n^{\prime}}|n\rangle\left\langle m^{\prime}\right| \\
& -\delta_{m n^{\prime}} \frac{1}{2}\left(\delta_{m^{\prime} k}|n\rangle\left\langle l\left|+\delta_{n l}\right| k\right\rangle\left\langle m^{\prime}\right|\right) .
\end{aligned}
$$

Assuming the spectrum of $\mathcal{U}_{S}$ is nondegenerate, the commutativity of $\widetilde{\mathcal{D}}$ and $\widetilde{\mathcal{H}}_{S}$ [Eq. (A8)] implies the eigenoperator condition

$$
\mathcal{D}^{*}\left[\hat{F}_{k}\right] \propto \hat{F}_{k} .
$$

This induces strict restrictions on the components $\mathcal{D}_{n m n^{\prime} m^{\prime}}^{*}$ which can appear in $\mathcal{D}^{*}$. To analyze which components contribute to $\mathcal{D}^{*}$, it is convenient to represent the dissipator in Liouville space. The elements of the superoperator in Liouville space in the basis $\mathfrak{F}$ are defined as $d_{i j}=\operatorname{tr}\left(\hat{S}_{i}^{\dagger} \mathcal{D}\left[\hat{S}_{j}\right]\right)$. Since $\{\hat{F}\}$ are eigenoperators of $\mathcal{D}^{*}, \widetilde{\mathcal{D}}^{*}$ obtains the form

$$
\widetilde{\mathcal{D}}^{*}=\left[\begin{array}{cc}
\tilde{\Delta}_{v} & 0 \\
0 & \tilde{\Delta}_{i}
\end{array}\right],
$$

where $\tilde{\Delta}_{\mathcal{V}}$ is diagonal and $\tilde{\Delta}_{i}$ is a diagonalizable matrix. The form of $\widetilde{\mathcal{D}}^{*}$ highlights that the unitary invariant and noninvariant components are independent,

$$
\mathcal{D}^{*}\left[\hat{\Pi}_{n}\right]=\sum_{n m} b_{n m} \hat{\Pi}_{m} .
$$

Relations (C4) and (C6) greatly restrict the term $\mathcal{D}_{n m n^{\prime} m}^{*}$ included in the dissipator. We analyze the different cases: For $n \neq m^{\prime}$ only the first term in Eq. (C2) contributes; condition (C4) then implies that $n=k$ and $m^{\prime}=l$. Hence, for $n \neq m^{\prime}$, only terms of the form $\mathcal{D}_{k k l l}^{*}$ contribute to $\mathcal{D}^{*}$. One can maybe suspect that somehow a combination of terms can cancel each other allowing for condition (C4) to be satisfied without nulling $\mathcal{D}_{n m n^{\prime} m^{\prime}}^{*}$ components which do not comply with Eqs. (C4) and (C6). However, this cannot be, since each component is associated with a different nondiagonal matrix element $d_{i j} \equiv d_{k l k^{\prime} l^{\prime}}=\operatorname{tr}\left(|l\rangle\langle k| \mathcal{D}\left[\left|k^{\prime}\right\rangle\left\langle l^{\prime}\right|\right]\right)$ with $k \neq k^{\prime}$ and $l \neq l^{\prime}$. When $m=n^{\prime}$ and $k=l$ (substituting the projection operator $\hat{\Pi}_{k}$ into $\mathcal{D}_{n m m m^{\prime}}$ ), Eq. (C6) infers that we must have $m=n^{\prime}$. Thus only terms of the form $\mathcal{D}_{k l l k}^{*}$ contribute to $\mathcal{D}^{*}$

Overall, the restrictions imply that only two possible components can contribute to the sums $\mathcal{D}_{k k l l}^{*}$ and $\mathcal{D}_{k l l k}^{*}$, leading to a restricted form of Eq. (C7). Substituting the transition operators $\hat{F}_{k}$ into $\mathcal{D}^{*}$ and demanding that the eigenoperator condition holds leads to the following master equation:

$$
\begin{aligned}
\mathcal{D}^{*}[\bullet]= & \sum_{k=-M}^{M} \gamma_{k}\left(\hat{F}_{k}^{\dagger} \bullet \hat{F}_{k}-\frac{1}{2}\left\{\hat{F}_{k}^{\dagger} \hat{F}_{k}, \bullet\right\}\right) \\
& +\sum_{i, j=1}^{N} \alpha_{i j}\left(\hat{\Pi}_{i} \bullet \hat{\Pi}_{j}-\frac{1}{2}\left\{\hat{\Pi}_{i} \hat{\Pi}_{j}, \bullet\right\}\right),
\end{aligned}
$$

where $M=N(N-1) / 2, \quad \hat{F}_{k}^{\dagger}=\hat{F}_{-k}, \quad$ and $\quad \gamma_{k}=a_{k k} \quad$ with $\gamma_{0}=0$.

\section{APPENDIX D: PROPERTIES OF THE MATRIX $\alpha$}

To study the properties of the matrix $\alpha$, we first analyze certain elements of the $r$ matrix. This is achieved by building upon the general construction presented in Appendix B.

We choose the orthonormal basis $\{\hat{S}\}$ to be composed of the transition operator of $\{\hat{F}\}$ and an orthonormal set $\{\hat{G}\}$ which spans the unitary invariant space of $\mathcal{U}_{S}$. Specifically, we choose the basis of the unitary invariant subspace $\{\hat{G}\}$ to be proportionate to the diagonal generalized Gell-Mann matrices [54]. As required, the Gell-Mann matrices are traceless, but may have to be renormalized. We therefore scale them so the basis $\mathfrak{F}$ has the same properties as $\{\hat{S}\}$ in Appendix B. The identification of $\{\hat{G}\}$ with the Gell-Mann matrices (up to a constant) allows us to express the unitary invariant basis operator in terms of the energy projection operators

$$
\hat{G}_{k}=\sum_{l} g_{k l} \hat{\Pi}_{l},
$$

where the weights $g_{k l}$ are real.

We utilize this relation to analyze the properties of coefficients $r_{i j}$. Inserting Eq. (D1) into the coefficients $r_{i j}$ [Eq. (B3)] associated with $\hat{S}_{i}, \hat{S}_{j} \in\{\hat{G}\}$ leads to

$$
\begin{aligned}
r_{G_{i} G_{j}} & =\sum_{\mu, \nu}\left(\hat{G}_{i}, \hat{K}_{\mu \nu}\right)\left(\hat{G}_{j}, \hat{K}_{\mu \nu}\right)^{*} \\
& =\sum_{l, l^{\prime}} g_{i l} g_{j l^{\prime}} \sum_{\mu, \nu}\left(\hat{\Pi}_{l}, \hat{K}_{\mu \nu}\right)\left(\hat{\Pi}_{l^{\prime}}, \hat{K}_{\mu \nu}\right)^{*} .
\end{aligned}
$$


Utilizing the inner product in Liouville space, the sum over inner products can be expressed as

$$
\begin{aligned}
\Xi & \equiv \sum_{\mu, \nu}\left(\hat{\Pi}_{l}, \hat{K}_{\mu \nu}\right)\left(\hat{\Pi}_{l^{\prime}}, \hat{K}_{\mu \nu}\right)^{*} \\
& =\sum_{\mu \nu}\left\langle l\left|\hat{K}_{\mu \nu}\right| l\right\rangle\left\langle l^{\prime}\left|\hat{K}_{\mu \nu}^{\dagger}\right| l^{\prime}\right\rangle .
\end{aligned}
$$

Substituting the explicit form of the Kraus operators $\hat{K}_{\mu \nu}=$ $\sqrt{\lambda_{\mu}}\left\langle\chi_{\nu}|\hat{U}(t, 0)| \chi_{\mu}\right\rangle[$ Eq. (A2)] leads to

$$
\begin{aligned}
\Xi & =\sum_{\mu \nu}\left\langle i, \chi_{\nu}|\hat{U}(t, 0)| \chi_{\mu}, i\right\rangle\left\langle j \chi_{\mu}|\hat{U}(t, 0)| \chi_{\nu}, j\right\rangle \\
& =\sum_{\nu}\left\langle i, \chi_{\nu}|\hat{U}(t, 0)| i\right\rangle\left(\sum_{\mu}\left|\chi_{\mu}\right\rangle\left\langle\chi_{\mu}\right|\right)\left\langle j|\hat{U}(t, 0)| \chi_{\nu}, j\right\rangle \\
& =\operatorname{tr}_{E}(\langle i|\hat{U}(t, 0)| i\rangle\langle j|\hat{U}(t, 0)| j\rangle)=\Xi^{*} .
\end{aligned}
$$

The third equality is implied by the completeness relation and the definition of the partial trace. The fourth equality stems from the unitarity of the propagator. Equation (D4) implies that $\Xi$ is real.

We can now conclude that the coefficient matrix of the unitary invariant subspace $r^{\text {inv }}=\left[r_{G_{i} G_{j}}\right]$ is a real Hermitian matrix, or equivalently, a symmetric matrix. As a result, the associated coefficient matrix $a^{\text {inv }}=\left[a_{G_{i} G_{j}}\right]$ [Eq. (B4)] is also symmetric. In the main text the coefficient matrix $\alpha$ is obtained by a basis transformation, from $\{\hat{G}\}$ to $\{\hat{\Pi}\}$; this implies that $\alpha$ can be diagonalized by a similarity transformation by means of an orthogonal matrix $Q: Q^{T} \alpha Q=\operatorname{diag}\left(\lambda_{1}, \ldots, \lambda_{N}\right)$. The property that the elements of $Q$ are real implies that any operator of the form $\hat{V}_{n}=\sum_{n} Q_{n i} \hat{\Pi}_{n}$ is Hermitian.

\section{APPENDIX E: DETAILED BALANCE BY MEANS OF THE GENERATOR'S FIXED POINT}

The fixed point of $\mathcal{L}$ determines a detailed balance relation between two dependent coefficients $\gamma_{k}$ and $\gamma_{-k}$, Eq. (C7). To show this property, we calculate the action of the generator on the fixed point and enforce the condition for which $\mathcal{L}\left[\hat{\rho}_{S}^{t h}\right]=$ 0 . The fixed point $\rho_{S}^{t h}=Z_{S}^{-1} e^{-\beta \hat{H}_{S}}$ trivially commutes with $\hat{H}_{S}$, which simplifies the invariance condition to $\mathcal{D}\left[\hat{\rho}_{S}^{\text {th }}\right]=0$.

The dissipative term is composed of two sums. The first sum includes the unitary noninvariant operators $\{\hat{F}\}$ of $\mathcal{U}_{S}$ and induces transitions between the energy levels. The second sum is composed of unitary invariant operators and generates pure dephasing. For simplicity we introduce a short notation for these sums, $\mathcal{D}=\mathcal{D}_{n}+\mathcal{D}_{i}$. The pure-dephasing component of the dissipator $\mathcal{D}_{i}$ is composed of energy projection operators $\{\hat{\Pi}\}$. With the help of the relation

$$
\hat{\Pi}_{k}^{\dagger} \hat{\Pi}_{r} \hat{\Pi}_{l}-\frac{1}{2}\left\{\hat{\Pi}_{k}^{\dagger} \hat{\Pi}_{l}, \hat{\Pi}_{r}\right\}=0,
$$

we can infer that $\mathcal{D}_{i}\left[\hat{\rho}_{S}^{\text {th }}\right]=0$. In turn, the invariance condition then becomes

$$
\mathcal{D}_{v}\left[e^{-\beta \hat{H}_{S}}\right]=0 .
$$

The Lindblad jump operators of $\mathcal{D}_{n}$ are transition operators between the energy levels of $\hat{H}_{S}$. This property implies that

$$
\left[\hat{H}_{S}, \hat{F}_{n m}\right]=-\hbar \omega_{n m} \hat{F}_{n m},
$$

where $\omega_{n m}=\left(\varepsilon_{m}-\varepsilon_{n}\right) / \hbar$ is the Bohr frequency between energy levels $|n\rangle$ and $|m\rangle$. Utilizing the commutation relations and the Baker-Campbell-Hausdorff formula, we deduce that

$$
\hat{F}_{n m} e^{-\beta \hat{H}_{S}}=e^{-\beta \hat{H}_{S}} \hat{F}_{n m} e^{-\beta \hbar \omega_{n m}} .
$$

We now utilize this relation to calculate $\mathcal{D}\left[e^{-\beta \hat{H}_{S}}\right]$ assuming general kinetic coefficients.

The $k$ th term of $\mathcal{D}_{v}$ is constructed from two terms $\mathcal{D}_{v}^{k}=$ $\mathcal{B}_{v}^{k}+\mathcal{B}_{v}^{-k}$ (recall that $k$ runs over double indices $n$ and $m$ ) with

$$
\mathcal{B}_{v}^{k}\left[e^{-\beta \hat{H}_{S}}\right]=\gamma_{k}\left(\hat{F}_{n m} e^{-\beta \hat{H}_{S}} \hat{F}_{n m}^{\dagger}-\frac{1}{2}\left\{\hat{F}_{n m}^{\dagger} \hat{F}_{n m}, e^{-\beta \hat{H}_{S}}\right\}\right)
$$

and

$$
\mathcal{B}_{v}^{-k}\left[e^{-\beta \hat{H}_{S}}\right]=\gamma_{-k}\left(\hat{F}_{n m}^{\dagger} e^{-\beta \hat{H}_{S}} \hat{F}_{n m}-\frac{1}{2}\left\{\hat{F}_{n m} \hat{F}_{n m}^{\dagger}, e^{-\beta \hat{H}_{S}}\right\}\right)
$$

With the help of relation (E4) and its Hermitian conjugate we find that

$$
\begin{aligned}
\mathcal{B}_{v}^{k}\left[e^{-\beta \hat{H}_{S}}\right] & =\gamma_{k}\left(e^{-\beta \omega_{n m}} \hat{F}_{n m} \hat{F}_{n m}^{\dagger}-\hat{F}_{n m}^{\dagger} \hat{F}_{n m}\right) e^{-\beta \hat{H}_{s}}, \\
\mathcal{B}_{v}^{-k}\left[e^{-\beta \hat{H}_{S}}\right] & =\gamma_{-k}\left(e^{\beta \omega_{n m}} \hat{F}_{n m}^{\dagger} \hat{F}_{n m}-\hat{F}_{n m} \hat{F}_{n m}^{\dagger}\right) e^{-\beta \hat{H}_{S}} .
\end{aligned}
$$

Since terms $\mathcal{D}_{v}^{k}$ and $\mathcal{D}_{v}^{k^{\prime}}$ are independent, condition (E2) translates to $N(N-1) / 2$ independent requirements

$$
\mathcal{D}_{v}^{k}\left[e^{-\beta \hat{H}_{S}}\right]=\mathcal{B}_{v}^{k}\left[e^{-\beta \hat{H}_{S}}\right]+\mathcal{B}_{v}^{-k}\left[e^{-\beta \hat{H}_{S}}\right]=0 .
$$

Inserting Eq. (E7) into condition (E8) leads to the detailed balance relation between the kinetic coefficients

$$
\gamma_{k}=\gamma_{-k} e^{\beta \omega_{n m}} \text {. }
$$

\section{APPENDIX F: COMMUTATIVITY OF DYNAMICAL MAPS AND STRICT ENERGY CONSERVATION CONDITION}

This Appendix analyzes the connection between commutativity of the open (Markovian) and isolated dynamical maps and the condition of strict energy conservation. Specifically, assuming commutation of the dynamical maps, we find the associated restrictions on the composite (environment and system) and system Hamiltonians.

Consider an open system with dynamics of a Markovian nature which originate from a general composite Hamiltonian $\hat{H}^{\prime}$ with an arbitrary interaction. In general, $\left[\hat{H}^{\prime}, \hat{H}_{S}\right]=\hat{X}$, for some global operator $\hat{X}$. The semigroup property then implies that $\Lambda_{n \tau}=\left(\Lambda_{\tau}\right)^{n}$, where $n \in \mathbb{N}$. This apparently cannot be correct for an arbitrary coarse-graining time $\tau$, since for small enough $\tau$ the Markovian assumption breaks. Therefore, in the present description, $\tau$ must be greater than (but on the order of) the timescale associated with the decay of correlations in the environment. Typically, in the Markovian regime the environment's intrinsic timescale is the fastest one: $\tau \ll \hbar /\left\|\hat{H}_{S}\right\|, \hbar /\left\|\hat{H}_{S E}\right\|$. Hence the total propagator can be approximated by

$$
\hat{U}_{S}(\tau, 0) \approx \hat{I}-\frac{i}{\hbar} \hat{H}_{S} \tau, \quad \hat{U}(\tau, 0) \approx \hat{I}-\frac{i}{\hbar} \hat{H}^{\prime} \tau .
$$

Utilizing these relations, the commutation relation of the "infinitesimal" maps becomes

$$
\begin{gathered}
\Lambda_{\tau}\left[\mathcal{U}_{S}(\tau, 0)[\hat{\rho}]\right]-\mathcal{U}_{S}(\tau, 0)\left[\Lambda_{\tau}[\hat{\rho}]\right] \\
=\Upsilon \tau^{3}+\Xi \tau^{4}+O\left(\tau^{5}\right),
\end{gathered}
$$


where the initial state is denoted by $\hat{\rho} \equiv \hat{\rho}_{S}(0) \otimes \hat{\rho}_{E}(0)$. The first- and second-order terms vanish, and the higher-order terms are given by

$$
\begin{aligned}
& \Upsilon=\frac{i}{\hbar^{3}} \operatorname{tr}_{E}\left(\left(\hat{H}^{\prime}+\hat{H}_{S}\right) \hat{\rho} \hat{X}-\hat{X} \hat{\rho}\left(\hat{H}^{\prime}+\hat{H}_{S}\right)\right), \\
& \Xi=\frac{1}{\hbar^{2}} \operatorname{tr}_{E}\left(\hat{X} \hat{\rho} \hat{H}^{\prime} \hat{H}_{S}-\hat{H}^{\prime} \hat{H}_{S} \hat{\rho} \hat{X}\right) .
\end{aligned}
$$

We keep track of terms up to fourth order in $\tau$ to be consistent with the linearization of the propagators, Eq. (F1). In the applied Markovian framework, the higherorder terms, $\Upsilon$ and $\Xi$, must vanish when the maps commute.
In turn, this imposes two strict conditions on the form of the general Hamiltonian $\hat{H}^{\prime}$, Eq. (F3). We find that if an arbitrary system state $\hat{\rho}_{S}(t)$ and a stationary environment state $\hat{\rho}_{E}$ are considered, except for pathological cases [that satisfy Eq. (F3)], the commutativity of the maps implies that $\hat{X}$ must vanish and strict energy conservation holds [98].

Overall, in the Markovian regime, excluding pathological cases, commutativity of $\Lambda_{t}$ and $\mathcal{U}_{S}(t, 0)$ implies that the composite dynamics are generated by a strict energy-conserving Hamiltonian. In addition, it reflects time-translational symmetry [99]. Hence there is a close connection between strict energy conservation and the commutativity property of the dynamical maps.
[1] R. Alicki and K. Lendi, Quantum Dynamical Semigroups and Applications, Lecture Notes in Physics Vol. 717 (Springer, New York, 2007).

[2] H.-P. Breuer and F. Petruccione, The Theory of Open Quantum Systems (Oxford University Press, New York, 2002).

[3] T. Albash, S. Boixo, D. A. Lidar, and P. Zanardi, Quantum adiabatic Markovian master equations, New J. Phys. 14, 123016 (2012).

[4] R. Alicki, The Markov master equations and the Fermi golden rule, Int. J. Theor. Phys. 16, 351 (1977).

[5] R. Alicki, D. Gelbwaser-Klimovsky, and G. Kurizki, Periodically driven quantum open systems: Tutorial, arXiv:1205.4552.

[6] H.-P. Breuer and B. Vacchini, Quantum Semi-Markov Processes, Phys. Rev. Lett. 101, 140402 (2008).

[7] S. Daffer, K. Wódkiewicz, J. D. Cresser, and J. K. McIver, Depolarizing channel as a completely positive map with memory, Phys. Rev. A 70, 010304(R) (2004).

[8] R. Dann, A. Levy, and R. Kosloff, Time-dependent Markovian quantum master equation, Phys. Rev. A 98, 052129 (2018).

[9] E. B. Davies, Markovian master equations, Commun. Math. Phys. 39, 91 (1974).

[10] L. Diósi, Calderia-Leggett master equation and medium temperatures, Physica A (Amsterdam) 199, 517 (1993).

[11] D. A. Lidar, Z. Bihary, and K. B. Whaley, From completely positive maps to the quantum Markovian semigroup master equation, Chem. Phys. 268, 35 (2001).

[12] C. Majenz, T. Albash, H.-P. Breuer, and D. A. Lidar, Coarse graining can beat the rotating-wave approximation in quantum Markovian master equations, Phys. Rev. A 88, 012103 (2013).

[13] S. Maniscalco and F. Petruccione, Non-Markovian dynamics of a qubit, Phys. Rev. A 73, 012111 (2006).

[14] G. McCauley, B. Cruikshank, D. I. Bondar, and K. Jacobs, Accurate Lindblad-form master equation for weakly damped quantum systems across all regimes, npj Quantum Inf. 6, 74 (2020).

[15] E. Mozgunov and D. Lidar, Completely positive master equation for arbitrary driving and small level spacing, Quantum 4, 227 (2020).

[16] C. Müller and T. M. Stace, Deriving Lindblad master equations with Keldysh diagrams: Correlated gain and loss in higher order perturbation theory, Phys. Rev. A 95, 013847 (2017).

[17] S. Nakajima, On quantum theory of transport phenomena: Steady diffusion, Prog. Theor. Phys. 20, 948 (1958).
[18] F. Nathan and M. S. Rudner, Universal Lindblad equation for open quantum systems, Phys. Rev. B 102, 115109 (2020).

[19] J. Piilo, S. Maniscalco, K. Härkönen, and K.-A. Suominen, Non-Markovian Quantum Jumps, Phys. Rev. Lett. 100, 180402 (2008).

[20] A. Shabani and D. A. Lidar, Completely positive postMarkovian master equation via a measurement approach, Phys. Rev. A 71, 020101(R) (2005).

[21] A. Y. Smirnov and M. H. Amin, Theory of open quantum dynamics with hybrid noise, New J. Phys. 20, 103037 (2018).

[22] K. Szczygielski, D. Gelbwaser-Klimovsky, and R. Alicki, Markovian master equation and thermodynamics of a two-level system in a strong laser field, Phys. Rev. E 87, 012120 (2013).

[23] R. S. Whitney, Staying positive: going beyond Lindblad with perturbative master equations, J. Phys. A: Math. Theor. 41, 175304 (2008).

[24] R. Zwanzig, Ensemble method in the theory of irreversibility, J. Chem. Phys. 33, 1338 (1960).

[25] R. Karplus and J. Schwinger, A note on saturation in microwave spectroscopy, Phys. Rev. 73, 1020 (1948).

[26] A. G. Redfield, On the theory of relaxation processes, IBM J. Res. Dev. 1, 19 (1957).

[27] R. K. Wangsness and F. Bloch, The dynamical theory of nuclear induction, Phys. Rev. 89, 728 (1953).

[28] R. Dümcke, The low density limit for an $N$-level system interacting with a free Bose or Fermi gas, Commun. Math. Phys. 97, 331 (1985).

[29] V. Gorini, A. Kossakowski, and E. C. G. Sudarshan, Completely positive dynamical semigroups of $N$-level systems, J. Math. Phys. (Melville, NY) 17, 821 (1976).

[30] G. Lindblad, On the generators of quantum dynamical semigroups, Commun. Math. Phys. 48, 119 (1976).

[31] K. Kraus, General state changes in quantum theory, Ann. Phys. (Amsterdam) 64, 311 (1971).

[32] G. Lindblad, Expectations and entropy inequalities for finite quantum systems, Commun. Math. Phys. 39, 111 (1974).

[33] A. Frigerio, Quantum dynamical semigroups and approach to equilibrium, Lett. Math. Phys. 2, 79 (1977).

[34] L. Accardi, A. Frigerio, and J. T. Lewis, Quantum stochastic processes, Publ. Res. Inst. Math. Sci. 18, 97 (1982).

[35] E. B. Davies, Quantum Theory of Open Systems (Academic, New York, 1976). 
[36] S. Milz, M. Kim, F. A. Pollock, and K. Modi, Completely Positive Divisibility Does Not Mean Markovianity, Phys. Rev. Lett. 123, 040401 (2019).

[37] D. Janzing, P. Wocjan, R. Zeier, R. Geiss, and T. Beth, Thermodynamic cost of reliability and low temperatures: Tightening Landauer's principle and the second law, Int. J. Theor. Phys. 39, 2717 (2000).

[38] F. G. Brandao, M. Horodecki, J. Oppenheim, J. M. Renes, and R. W. Spekkens, Resource Theory of Quantum States Out of Thermal Equilibrium, Phys. Rev. Lett. 111, 250404 (2013).

[39] M. Lostaglio, An introductory review of the resource theory approach to thermodynamics, Rep. Prog. Phys. 82, 114001 (2019).

[40] N. Megier, A. Smirne, and B. Vacchini, The interplay between local and non-local master equations: Exact and approximated dynamics, New J. Phys. 22, 083011 (2020).

[41] M. Lostaglio, D. Jennings, and T. Rudolph, Description of quantum coherence in thermodynamic processes requires constraints beyond free energy, Nat. Commun. 6, 6383 (2015).

[42] M. Lostaglio, K. Korzekwa, D. Jennings, and T. Rudolph, Quantum Coherence, Time-Translation Symmetry, and Thermodynamics, Phys. Rev. X 5, 021001 (2015).

[43] F. Brandao, M. Horodecki, N. Ng, J. Oppenheim, and S. Wehner, The second laws of quantum thermodynamics, Proc. Natl. Acad. Sci. USA 112, 3275 (2015).

[44] M. Horodecki and J. Oppenheim, Fundamental limitations for quantum and nanoscale thermodynamics, Nat. Commun. 4, 2059 (2013)

[45] V. V. Albert, B. Bradlyn, M. Fraas, and L. Jiang, Geometry and Response of Lindbladians, Phys. Rev. X 6, 041031 (2016).

[46] R. Alicki, On the detailed balance condition for nonHamiltonian systems, Rep. Math. Phys. 10, 249 (1976).

[47] U. Fano, Description of states in quantum mechanics by density matrix and operator techniques, Rev. Mod. Phys. 29, 74 (1957).

[48] I. Gohberg, S. Goldberg, and M. A. Kaashoek, Hilbert-Schmidt operators, in Classes of Linear Operators (Springer, New York, 1990), Vol. I, pp. 138-147.

[49] M. Sarandy and D. Lidar, Adiabatic approximation in open quantum systems, Phys. Rev. A 71, 012331 (2005).

[50] M. Sarandy and D. Lidar, Abelian and non-Abelian geometric phases in adiabatic open quantum systems, Phys. Rev. A 73, 062101 (2006).

[51] Commutativity of $\mathcal{H}_{S}$ and $\mathcal{L}=i \mathcal{H}+\mathcal{D}$ implies that the associated superoperators in Hilbert-Schmidt space satisfy $i\left[\widetilde{\mathcal{H}}_{S}, \widetilde{\mathcal{H}}\right]=-\left[\widetilde{\mathcal{H}}_{S}, \widetilde{\mathcal{D}}\right]$. Such a case is pathological and does not occur regularly.

[52] H. A. Bethe, The electromagnetic shift of energy levels, Phys. Rev. 72, 339 (1947).

[53] W. E. Lamb Jr. and R. C. Retherford, Fine structure of the hydrogen atom by a microwave method, Phys. Rev. 72, 241 (1947).

[54] R. A. Bertlmann and P. Krammer, Bloch vectors for qudits, J. Phys. A: Math. Theor. 41, 235303 (2008).

[55] M. Gell-Mann, Symmetries of baryons and mesons, in Murray Gell-Mann: Selected Papers (World Scientific, Singapore, 2010), pp. 128-145.

[56] H. Georgi, Lie Algebras in Particle Physics: From Isospin to Unified Theories (CRC, Boca Raton, 2018).
[57] G. Bulnes Cuetara, M. Esposito, and G. Schaller, Quantum thermodynamics with degenerate eigenstate coherences, Entropy 18, 447 (2016).

[58] B. B. Laird, J. Budimir, and J. L. Skinner, Quantum-mechanical derivation of the Bloch equations: Beyond the weak-coupling limit, J. Chem. Phys. 94, 4391 (1991).

[59] J. O. González, L. A. Correa, G. Nocerino, J. P. Palao, D. Alonso, and G. Adesso, Testing the validity of the 'local' and 'global' GKLS master equations on an exactly solvable model, Open Syst. Inf. Dyn. 24, 1740010 (2017).

[60] P. P. Hofer, M. Perarnau-Llobet, L. D. M. Miranda, G. Haack, R. Silva, J. B. Brask, and N. Brunner, Markovian master equations for quantum thermal machines: Local versus global approach, New J. Phys. 19, 123037 (2017).

[61] A. Rivas, A. D. K. Plato, S. F. Huelga, and M. B. Plenio, Markovian master equations: A critical study, New J. Phys. 12, 113032 (2010).

[62] G. Lindblad, On the existence of quantum subdynamics, J. Phys. A: Math. Gen. 29, 4197 (1996).

[63] A. Ishizaki and Y. Tanimura, Quantum dynamics of system strongly coupled to low-temperature colored noise bath: Reduced hierarchy equations approach, J. Phys. Soc. Jpn. 74, 3131 (2005).

[64] L. Li, M. J. Hall, and H. M. Wiseman, Concepts of quantum non-Markovianity: A hierarchy, Phys. Rep. 759, 1 (2018).

[65] J. M. Dominy and D. A. Lidar, Beyond complete positivity, Quantum Inf. Process. 15, 1349 (2016).

[66] P. Pechukas, Reduced Dynamics Need Not Be Completely Positive, Phys. Rev. Lett. 73, 1060 (1994).

[67] R. Alicki, Comment on "Reduced Dynamics Need Not Be Completely Positive”, Phys. Rev. Lett. 75, 3020 (1995).

[68] H. Spohn and J. L. Lebowitz, Irreversible thermodynamics for quantum systems weakly coupled to thermal reservoirs, Adv. Chem. Phys. 38, 109 (1978).

[69] R. Hartmann and W. T. Strunz, Accuracy assessment of perturbative master equations: Embracing nonpositivity, Phys. Rev. A 101, 012103 (2020).

[70] R. Bulla, T. A. Costi, and T. Pruschke, Numerical renormalization group method for quantum impurity systems, Rev. Mod. Phys. 80, 395 (2008).

[71] R. Alicki and M. Fannes, Quantum Dynamical Systems (Oxford University Press, Oxford, 2001).

[72] L. D’Alessio, Y. Kafri, A. Polkovnikov, and M. Rigol, From quantum chaos and eigenstate thermalization to statistical mechanics and thermodynamics, Adv. Phys. 65, 239 (2016).

[73] J. M. Deutsch, Eigenstate thermalization hypothesis, Rep. Prog. Phys. 81, 082001 (2018).

[74] M. Rigol, V. Dunjko, and M. Olshanii, Thermalization and its mechanism for generic isolated quantum systems, Nature (London) 452, 854 (2008).

[75] M. Srednicki, Chaos and quantum thermalization, Phys. Rev. E 50, 888 (1994).

[76] H. De Raedt, F. Jin, M. I. Katsnelson, and K. Michielsen, Relaxation, thermalization, and Markovian dynamics of two spins coupled to a spin bath, Phys. Rev. E 96, 053306 (2017).

[77] I. Burghardt, M. Nest, and G. A. Worth, Multiconfigurational system-bath dynamics using Gaussian wave packets: Energy 
relaxation and decoherence induced by a finite-dimensional bath, J. Chem. Phys. 119, 5364 (2003).

[78] V. Vadimov, J. Tuorila, T. Orell, J. Stockburger, T. AlaNissila, J. Ankerhold, and M. Mottonen, Validity of BornMarkov master equations for single and two-qubit systems, arXiv:2011.05046.

[79] F. Barra, The thermodynamic cost of driving quantum systems by their boundaries, Sci. Rep. 5, 14873 (2015).

[80] A. Levy and R. Kosloff, The local approach to quantum transport may violate the second law of thermodynamics, Europhys. Lett. 107, 20004 (2014).

[81] A. Hewgill, G. De Chiara, and A. Imparato, Quantum thermodynamically consistent local master equations, Phys. Rev. Research 3, 013165 (2021).

[82] S. Scali, J. Anders, and L. A. Correa, Local master equations bypass the secular approximation, arXiv:2009.11324.

[83] G. De Chiara, G. Landi, A. Hewgill, B. Reid, A. Ferraro, A. J. Roncaglia, and M. Antezza, Reconciliation of quantum local master equations with thermodynamics, New J. Phys. 20, 113024 (2018).

[84] M. Cattaneo, G. L. Giorgi, S. Maniscalco, and R. Zambrini, Local versus global master equation with common and separate baths: superiority of the global approach in partial secular approximation, New J. Phys. 21, 113045 (2019).

[85] M. Cattaneo, G. L. Giorgi, S. Maniscalco, and R. Zambrini, Symmetry and block structure of the Liouvillian superoperator in partial secular approximation, Phys. Rev. A 101, 042108 (2020)

[86] D. Farina and V. Giovannetti, Open-quantum-system dynamics: Recovering positivity of the Redfield equation via the partial secular approximation, Phys. Rev. A 100, 012107 (2019).

[87] G. Schaller and T. Brandes, Preservation of positivity by dynamical coarse graining, Phys. Rev. A 78, 022106 (2008).
[88] L. A. Correa, J. P. Palao, G. Adesso, and D. Alonso, Performance bound for quantum absorption refrigerators, Phys. Rev. E 87, 042131 (2013).

[89] N. Brunner, M. Huber, N. Linden, S. Popescu, R. Silva, and P. Skrzypczyk, Entanglement enhances cooling in microscopic quantum refrigerators, Phys. Rev. E 89, 032115 (2014).

[90] M. Am-Shallem, R. Kosloff, and N. Moiseyev, Exceptional points for parameter estimation in open quantum systems: Analysis of the Bloch equations, New J. Phys. 17, 113036 (2015).

[91] W. Heiss and A. Sannino, Avoided level crossing and exceptional points, J. Phys. A: Math. Gen. 23, 1167 (1990).

[92] N. Moiseyev, Non-Hermitian Quantum Mechanics (Cambridge University Press, Cambridge, 2011).

[93] N. Moiseyev and S. Friedland, Association of resonance states with the incomplete spectrum of finite complex-scaled Hamiltonian matrices, Phys. Rev. A 22, 618 (1980).

[94] M. Esposito, K. Lindenberg, and C. Van den Broeck, Entropy production as correlation between system and reservoir, New J. Phys. 12, 013013 (2010).

[95] J. Goold, M. Huber, A. Riera, L. Del Rio, and P. Skrzypczyk, The role of quantum information in thermodynamicstopical review, J. Phys. A: Math. Theor. 49, 143001 (2016).

[96] G. Lindblad, Entropy, information and quantum measurements, Commun. Math. Phys. 33, 305 (1973).

[97] R. Dann and R. Kosloff, Thermodynamically consistent dynamics of driven open quantum systems: from an autonomous framework to the semi-classical description, arXiv:2012.07979.

[98] Note that for a general system state $\hat{\rho}_{S}(0)$ the two terms in $\Upsilon$ may lead to different system operators, meaning that to satisfy the equality in Eq. (F3) both contributions must vanish.

[99] I. Marvian and R. W. Spekkens, Extending Noether's theorem by quantifying the asymmetry of quantum states, Nat. Commun. 5, 3821 (2014). 